\title{
COMPARACIÓN DE LOS NIVELES DE FLUOROSIS DENTAL EN ESCOLARES DE DOS MUNICIPIOS DE SANTANDER
}

\author{
${ }^{1}$ Horacio Blanco A., ${ }^{1}$ Lizzeth Durán A., ${ }^{1}$ Linda Nicole Neira R., ${ }^{1}$ Leyla Pourgoshtasbi V., ${ }^{1}$ Leidy Constanza Carvajal P., \\ ${ }^{2}$ Sonia Constanza Concha $S$. \\ ${ }^{1}$ Estudiante de $\mathrm{X}$ semestre F. de Odontología U. Santo Tomás. \\ ${ }^{2}$ Odontóloga U. Santo Tomás, Especialista en Educación y Comunicación para la Salud, MSc Epidemiología \\ $U$. Industrial de Santander, Docente U. Santo Tomás
}

Autor responsable de correspondencia: Horacio Blanco A.

Correo electrónico: hoblaan182@hotmail.com

\section{RESUMEN}

Objetivo: Describir los niveles de fluorosis dental en escolares entre los 6 y 17 años de edad vinculados a dos instituciones educativas de dos municipios de Santander.

Materiales y métodos: Se realizó un estudio observacional descriptivo de corte transversal; el universo y la muestra estuvo integrada por la totalidad de escolares vinculados a dos concentraciones educativas de dos municipios de Santander. Se recolectaron variables sociodemográficas, condiciones sistémicas, percepción de los menores frente a la apariencia de sus dientes, caries dental (índice COP) y la fluorosis dental (índice de Dean e índice de Thysltrup \& Fejerskov). El análisis estadístico implicó el cálculo de medidas de resumen según la naturaleza de las variables; para el bivariado se consideró como variable de salida, municipio de residencia y las otras como explicatorias, para las variables cualitativas se aplicó test de $\mathrm{Chi}^{2}$ y Exacto de Fisher y para las cuantitativas prueba t de Student o test de rangos de Wilcoxon.

Resultados: Se evaluaron 342 escolares, 249 sujetos procedían de Oiba y 93 de Cepitá. El 80.7\% de las personas del primer municipio y el $97.8 \%$ del segundo registraron fluorosis dental.

Conclusión: Aunque la prevalencia de fluorosis observada en el municipio de Cepitá fue superior a la de Oiba, los resultados deben mirarse con discreción debido a las diferencias de edad entre las poblaciones. [Blanco H, Durán L, Neira LN, Pourgoshtasbi L, Carvajal LC, Concha SC. Comparación de los niveles de fluorosis dental en escolares de dos municipios de Santander. Ustasalud 2008; 7: 108 - 116]

Palabras clave: Fluorosis, Prevalencia, Índice de Dean, Índice de Thysltrup \& Fejerskov, Escolares.

\section{COMPARISON OF THE LEVELS OF DENTAL FLUOROSIS IN STUDENTS OF TWO MUNICIPALITIES FROM SANTANDER}

\section{ABSTRACT}

Objective: To compare the levels of dental fluorosis in students between the 6 and 17 years old in two scholar institutions from two municipalities of Santander.

Material and methods: An observational descriptive cross sectional study was done; the universe and the sample were integrated by the totality of scholars of two educational institutions for two municipalities of Santander. Sociodemographic variables, systemic conditions, perception of the teeth appearance, dental decay (COP index) and dental flurosis (Dean and Thysltrup \& Fejerskov index) were registered. The statistic analysis implied the calculation of summary measures according to the nature of the variables; by bivariate analysis the municipality was considered like exit variable, for the qualitative variables Chi square and Fisher Exact test was applied and by the quantitative variables $t$ student and Wilcoxon ranks test was used.

Results: Three hundred forty and two students were evaluated, 249 subjects came from Oiba and 93 from Cepita. In Oiba, 80.7\% subjects and $97.8 \%$ from Cepita registered dental fluorosis.

Conclusions: Although the prevalence of fluorosis observed in Cepita were superior, the results must be look with discretion based on the differences of age between the two populations.

Key words: Dental fluorosis, Prevalence, Dean index, Thysltrup \& Fejerskov index, Scholars.

Recibido para publicación: 29 de septiembre de 2008. Aceptado para publicación: 14 de enero de 2009. 


\section{INTRODUCCIÓN}

La fluorosis dental, definida como una alteración dental asociada a la intoxicación crónica con fluoruros durante el periodo de gestación y a lo largo de los periodos de desarrollo del diente, ha registrado un aparente incremento en su frecuencia; este fenómeno se ha hecho evidente tanto a nivel nacional como internacional. ${ }^{1-3}$ Es probable que tal comportamiento se relacione con el mayor interés que ha mostrado la profesión odontológica frente al fenómeno.

Sin embargo, no se puede desconocer que esta condición se evidencia en niños y adolescentes en diferentes países en forma variable al punto que se han registrado reportes de prevalencia que oscilan entre el $20 \%$ y el $90 \%$. $^{1,3,4}$,

En el ámbito nacional, las políticas de fluoruración de la sal y la fluorosis dental han despertado interés y han generado controversia. Esto debería constituirse en una buena base para que el Estado y el Gremio Odontológico asuman una visión clara del evento, fundamentados en el comportamiento real de la fluorosis. Por lo tanto, sería necesario implementar acciones orientadas a promover la vigilancia epidemiológica de esta alteración del esmalte dental. Pues, como lo expresan Martínez y colaboradores se requiere el reconocimiento, desde una perspectiva socio-crítica de la importancia de la vigilancia epidemiológica de las enfermedades bucales (dentro de las que cabría considerar la fluorosis dental), los factores de riesgo y los determinantes para el seguimiento de los programas comunitarios en salud bucal, gestados desde el empoderamiento comunitario y con el fortalecimiento del gremio. ${ }^{5}$

La vigilancia en salud pública se puede definir como el seguimiento, recolección sistemática, análisis e interpretación de datos sobre eventos de salud o aspectos relacionados y que son utilizados en la planificación, ejecución y evaluación de los programas de salud pública. Un fundamento básico de la vigilancia es la divulgación de la información a las personas que la necesitan. ${ }^{6}$

Los mecanismos de captura de la información en los procesos de vigilancia epidemiológica deben fundamentarse en indicadores válidos y reproducibles, aplicables a grandes poblaciones y que sean costo efectivos lo que fundamenta la importancia de utilizar indicadores validados dentro de los que cabría destacar el índice de Dean y el índice de Thysltrup \& Fejerskov (TFI), este último en particular se soporta en la correlación histológica para la definición de cada uno de los criterios que lo definen. ${ }^{7}$ Sin embargo, es importante reconocer que el índice de Dean ha sido ampliamente utilizado en investigaciones a nivel nacional y mundial que sigue los parámetros establecidos por la Organización Mundial y Panamericana de la Salud (OMS/OPS) para la medición de este evento. ${ }^{1,8}$

Desde el 2000 aproximadamente, Tovar llama la atención sobre la importancia de implantar estrategias de vigilancia epidemiológica orientadas a monitorear la fluorosis dental. ${ }^{9}$ En la resolución 3577 de septiembre de 2006 por la cual se adoptaba el Plan Nacional de Salud Bucal-PNSB, se evidenció el interés del Estado por definir y desarrollar estrategias para el uso de la información y modelos de vigilancia, que soporten los logros e identifiquen oportunamente las acciones a seguir en términos de salud oral. ${ }^{10}$ Por otra parte, en el decreto 3039 de 10 de agosto de 2007 por el que se adoptaba el Plan Nacional de Salud Pública, se propuso como una de las estrategias para mejorar la salud oral, hacer vigilancia de la estrategia de fluoruración de la sal y fortalecer las acciones de vigilancia, prevención y control de la fluorosis. ${ }^{11}$ Sin embargo, en la resolución 0425 del 11 de febrero de 2008 por la cual se define el plan nacional de salud pública para intervenciones colectivas, no se evidencia ninguna acción específica orientada a responder a acciones de vigilancia de la fluorosis dental, ${ }^{12}$ lo que parece indicar la pérdida del interés del Estado frente al seguimiento y control de esta alteración.

Los reportes informales del público en general y de odontólogos parecen indicar que el fenómeno de fluorosis se evidencia con mayor frecuencia en la actualidad; hecho que se hizo manifiesto en Oiba, Santander. Llama la atención además que, a pesar de que en la literatura se reportan a diferentes municipios, entre ellos Cepitá, como lugares con potencial endemia de fluorosis, pues las fuentes de agua de consumo humano registran altos niveles de flúor (superan las 0,5 ppm); no se encontró en la literatura revisada, ningún estudio de base epidemiológica que permita soportar esta afirmación, pues un reporte encontrado, refería que Thomas Marthaler, asesor de la OPS/OMS encontró niveles de fluorosis leve para los municipios de Cepitá, Luruaco, Hobo y Gigante. ${ }^{13}$ Es importante anotar que la potencial exposición a los fluoruros entre estas dos poblaciones es diferente lo que permitiría suponer que el comportamiento de la fluorosis podría no ser igual, siendo superior en Cepitá; sin embargo, se carece de evidencia verificable que pueda sustentar esta afirmación.

Por lo tanto, objetivo del presente trabajo fue describir los niveles de fluorosis dental en escolares entre los 6 y 17 años de edad vinculados a dos instituciones educativas de dos municipios de Santander. 


\section{MATERIALES Y MÉTODOS}

Se realizó un estudio observacional descriptivo de corte transversal. ${ }^{14,15}$ El universo y la muestra estuvo integrado por 249 escolares de ambos géneros vinculados a una institución educativa pública de Oiba y 93 estudiantes de una concentración escolar de Cepitá, las dos en el departamento de Santander. Los criterios de inclusión definidos para el estudio fueron: haber nacido y proceder de los municipios objeto del estudio, estar entre los 6 y 17 años de edad, estar matriculados en las escuelas urbanas, estar presente en al escuela el día de la recolección de los datos, ser un menor de edad que presente dentición mixta y/o permanente.

Se excluyeron los escolares con compromiso sistémico y metabólico que afectara la apariencia clínica del esmalte, como alteraciones renales y/o gástricas; escolares con alteraciones mentales, con aparatología oral que impidiera la observación clínica y niños cuyos padres o ellos mismos se rehusaran a participar en el estudio.

En el momento del examen se excluyeron los dientes cariados, fracturados y obturados en más de las dos terceras partes de la superficie vestibular, dientes con cálculos en más de dos terceras partes de esta superficie y dientes con menos de $4 \mathrm{~mm}$ de corona clínica erupcionada.

Se recolectaron variables sociodemográficas como edad, género, y estrato socioeconómico; se interrogó a los padres sobre aspectos relacionadas con alteraciones sistémicas que afectaran al niño y a los escolares, sobre su percepción frente a la apariencia de los dientes. Se evalúo clínicamente el nivel de caries analizado mediante el índice COP y nivel fluorosis dental medidas de acuerdo a los criterios definidos para los índices de Dean y TFI. ${ }^{7,8}$

Previo al examen clínico, se realizó una prueba piloto en la que se estandarizaron cinco evaluadores y se aplicaron los criterios establecidos en Dental Fluorosis. A Handbook for Health Worker, ${ }^{7}$ bajo la dirección de un profesional entrenado en fluorosis. Se hizo énfasis en el diagnóstico diferencial fundamentado en el área dental afectada, forma, delimitación, color, dientes involucrados y otras pigmentaciones no fluoróticas. Los niveles de reproducibilidad alcanzaron niveles de Kappa de 0,64 a 0,72.

Para acceder a la población objeto de estudio, se concertó el trabajo con los directores de las instituciones educativas, quienes convocaron a los padres momento en el cual se explicaron los objetivos, los procedimientos y el alcance del estudio. Se obtuvo el consentimiento por parte de los padres y del escolar. Se aplicó una encuesta de autodiligenciamien- to dirigida a los padres de familia con el fin de saber el estado de salud tanto físico como mental de los niños y se interrogó sobre los aspectos considerados en la investigación.

El examen se llevó a cabo en las escuelas seleccionadas de la siguiente manera: a los escolares se le realizó la limpieza de los dientes por medio de cepillado manual en los baños del plantel educativo, luego los niños fueron llevados a un espacio bien iluminado y se sentaron en una silla donde el examinador se ubicó de frente al niño, se secaron los dientes con rollos de algodón. El examen intraoral se realizó por medio de la inspección visual bajo luz natural y un espejo de boca; finalmente, los resultados fueron registrados en un formulario para cada individuo por cada examinador, se anotó el examen para COP, DEAN, TFI. ${ }^{7,8}$

Los formatos ya diligenciados se recopilaron en un archivo; la base de datos se sistematizó en Excel por duplicado, se exportó al programa Epi-info 6.04 y mediante la rutina validate se verificó la calidad de la digitación. Posteriormente, se exportó al paquete estadístico STATA 9.0 para su correspondiente procesamiento y análisis. ${ }^{16-18}$

El plan de análisis estadístico implicó un análisis univariado en el que se describieron cada una de las variables en estudio mediante medidas de resumen de acuerdo con la naturaleza de las variables. ${ }^{19,20}$

Un análisis bivariado en que se consideró como variable de salida municipio de residencia y como variables explicatorios los otros criterios incluidos en el estudio; para esto se aplicaron test de Chi cuadrado o Exacto de Fisher cuando las variables eran de naturaleza cualitativa y prueba $t$ de Student o test de rangos de Wilcoxon cuando eran de naturaleza cuantitativa. ${ }^{19,20}$ Todo el análisis consideró un nivel de significancia de alfa $(\alpha)=0,05$

La presente investigación se acogió a la reglamentación establecida en la Resolución 008430 de 1993, en la que se respetó en los escolares los principios de beneficencia, autonomía, confidencialidad y privacidad de la información recolectada. ${ }^{21}$

\section{RESULTADOS}

\section{Análisis univariado}

Se evaluaron 342 escolares con un promedio de edad de 9,5 $\pm 2,8$ años; el 55,6\% (198) de los sujetos eran del género femenino; el 47,7\% (163) eran de estrato socioeconómico 1 y el 91,2\% (312) no registraba alteraciones sistémicas (Tabla 1).

El 85,4\% (292) registraban fluorosis y el promedio de dientes afectados con esta condición fue de $9.0 \pm 8,1$. 
El 23,4\% de los escolares reportaron no estar satisfechos con la apariencia de sus dientes (Tabla 2).

El promedio del COP fue de 3,4 $\pm 3,2$ dientes cariados, obturados y/o perdidos. El componente de cariados fue el que más le aportó al índice con una media de 2,4 2 2,6 (Tabla 3).

\section{Análisis bivariado}

En cuanto a las variables sociodemográficas se observaron asociaciones estadísticamente significativas para las variables edad y estrato socioeconómico, ambas con niveles de $\mathrm{p}<0,001$. Es importante anotar para edad, que mientras el promedio de edad de los escolares de Oiba fue de $8,8 \pm 1,8$, en Cepitá fue 12 $\pm 3,6$ años (Tabla 1).

Para las variables relacionadas con presencia de fluorosis, promedio de dientes con fluorosis y satisfacción frente a la apariencia de los dientes también se observaron niveles de $\mathrm{p}<0.001$; se observó mayor prevalencia de fluorosis $(97,8 \%)$ en los escolares de Cepitá comparado con los de Oiba $(80,7 \%)$. Mayor insatisfacción $(67,7 \%)$ frente a la apariencia de los dientes en los escolares de Cepitá comparado con los de Oiba (6,8\%). Finalmente, el promedio de dientes con fluorosis fue dos veces mayor en los escolares de Cepitá (Tabla 2).

Tabla 1. Descripción de las variables sociodemográficas y clínicas de los escolares evaluados analizadas en forma global y según el municipio de residencia.

\begin{tabular}{|c|c|c|c|c|}
\hline Variable & $\begin{array}{c}\text { Global } \\
\text { Frec (\%) }\end{array}$ & $\begin{array}{c}\text { Oiba } \\
\text { Frec (\%) }\end{array}$ & $\begin{array}{c}\text { Cepitá } \\
\text { Frec (\%) }\end{array}$ & $\mathbf{P}^{*}$ \\
\hline Global & $342(100)$ & $249(72.8)$ & $93(27.2)$ & --- \\
\hline Edad $†$ & $9,5 \pm 2,8$ & $8,8 \pm 1,8$ & $12 \pm 3,6$ & $<0,001^{* *} \dagger$ \\
\hline \multicolumn{5}{|l|}{ Sexo } \\
\hline - $\quad$ Femenino & $198(55,6)$ & $134(53,8)$ & $56(60,2)$ & $0,289^{* * *}$ \\
\hline - $\quad$ Masculino & $152(44,4)$ & $115(46,2)$ & $37(39,8)$ & \\
\hline \multicolumn{5}{|l|}{ Estrato socioeconómico } \\
\hline-1 & $163(47,7)$ & $75(30,1)$ & $88(94,6)$ & $<0,001 \dagger$ \\
\hline-2 & $152(44,4)$ & 149 (598) & $3(3,2)$ & \\
\hline 3 & $26(7,6)$ & $24(9,6)$ & $2(2,2)$ & \\
\hline-4 & $1(0,3)$ & $1(0,4)$ & --- & \\
\hline \multicolumn{5}{|l|}{ Alteraciones sistémicas } \\
\hline - $\quad$ Ninguna & $312(91,2)$ & $223(89,6)$ & $89(95,7)$ & 0,146 \\
\hline - Respiratorias & $26(7,6)$ & $23(9,2)$ & $3(3,2)$ & \\
\hline
\end{tabular}

* Test exacto de Fisher $\quad{ }^{* *}$ Prueba t de Student $\quad{ }^{* * *}$ Test de chi cuadrado $\quad \dagger$ Promedio $\pm \mathrm{DE} \quad \alpha=0,05$

Tabla 2. Descripción de las variables relacionadas con la presencia de fluorosis y potenciales factores asociados, analizadas en forma global y según el municipio de residencia.

\begin{tabular}{|c|c|c|c|c|}
\hline Variable & $\begin{array}{c}\text { Global } \\
\text { Frec (\%) }\end{array}$ & $\begin{array}{c}\text { Oiba } \\
\text { Frec (\%) }\end{array}$ & $\begin{array}{c}\text { Cepitá } \\
\text { Frec (\%) }\end{array}$ & $\mathbf{P}^{*}$ \\
\hline Global & $342(100)$ & $249(72.8)$ & $93(27.2)$ & --- \\
\hline Fluorosis & $292(85,4)$ & $201(80,7)$ & $91(97,8)$ & $<0,001$ \\
\hline Dientes con fluorosis $\dagger$ & $9 \pm 8,1$ & $7,2 \pm 6,2$ & $14 \pm 10,2$ & $<0,001^{* *}$ \\
\hline \multicolumn{5}{|l|}{ Apariencia dientes } \\
\hline Insatisfecho & $80(23,4)$ & $17(6,8)$ & $63(67,7)$ & $<0,001^{* * *}$ \\
\hline Satisfecho & $262(76,6)$ & $232(93,1)$ & $30(32,3)$ & \\
\hline
\end{tabular}

${ }^{*}$ Test exacto de Fisher $\quad{ }^{* *}$ Prueba $t$ de Student $\quad{ }^{* * *}$ Test de chi cuadrado $\quad \nmid$ Promedio $\pm \mathrm{DE} \quad \alpha=0,05$ 
Tabla 3. Relación de historia de caries (COP) analizada según la presencia de fluorosis y el municipio estudiado.

\begin{tabular}{lcccccc}
\hline \multicolumn{1}{c}{ Historia de caries } & \multicolumn{3}{c}{ Oiba } & \multicolumn{3}{c}{ Cepitá } \\
& Global & Si Fluorosis & No Fluorosis & Global & Si Fluorosis & No Fluorosis \\
\hline COP* $^{*}$ & $3,4 \pm 3,2$ & $3,3 \pm 3$ & $3,9 \pm 3,6$ & $3,1 \pm 2,8$ & $3,2 \pm 2,7$ & $2 \pm 2,8$ \\
Cariados* $^{*}$ & $2,4 \pm 2,6$ & $2,3 \pm 3$ & $3 \pm 2,9$ & $2,6 \pm 2,6$ & $2,7 \pm 2,6$ & $0,5 \pm 0,7$ \\
bbturados* $_{\text {Perdidos* }}$ & $0,6 \pm 1,2$ & $0,7 \pm 1,3$ & $0,5 \pm 1$ & $0,4 \pm 0,3$ & $0,4 \pm 0,8$ & $1,5 \pm 2,1$ \\
\hline
\end{tabular}

${ }^{*}$ Promedio $\pm \mathrm{DE}$

En cuanto a la caries dental, se observó que el promedio de caries dental en los escolares de Oiba que registraban fluorosis fue de 2,3 y de los no registraban fluorosis era de 2,6 ; en estos mismos grupos, en Cepitá fue de 2,7 y 0,5 , respectivamente. Este último resultado es importante analizarlo con cautela, ya que sólo dos escolares de Cepitá no registraron fluorosis.
En las Figuras 1 y 2 se evidencian como los niveles catalogados según el índice de Dean como esmalte normal y fluorosis dudosa, y los niveles de fluorosis de acuerdo al TFI catalogados de 0 a 2 predominaron en el municipio de Oiba. En Cepitá, se hicieron más evidentes las formas más severas tanto con el índice de Dean como con el TFI.

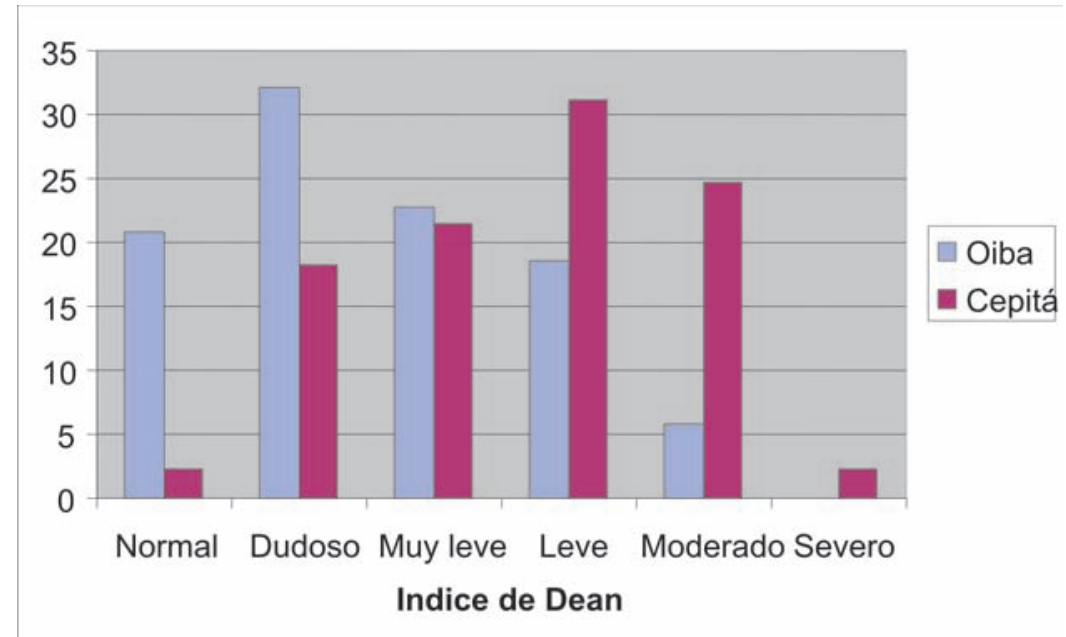

Figura 1. Descripción de los niveles de fluorosis según el índice de Dean de acuerdo con el municipio estudiado.

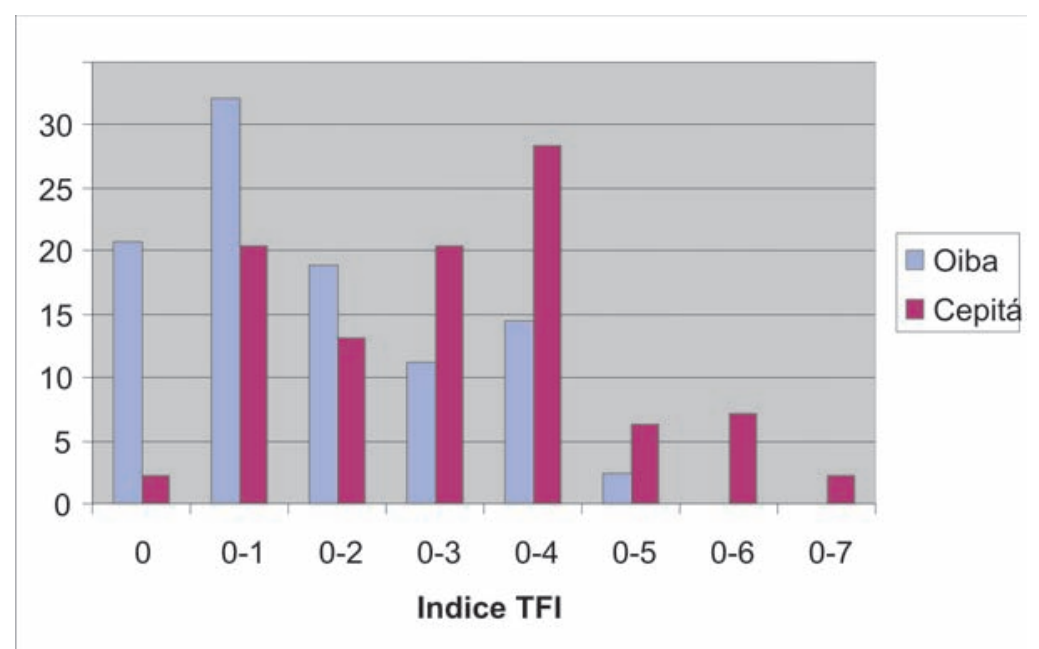

Figura 2. Descripción de los niveles de fluorosis según el índice TFI de acuerdo con el municipio estudiado. 


\section{Análisis por estructura y hemiarcada}

Las Figuras 3, 4, 5 y 6 permiten evidenciar el comportamiento de la fluorosis por hemiarcada y en cada estructura dentaria según el índice de Dean y el TFI; es importante resaltar los patrones de bilateralidad y similitudes en el comportamiento de la fluorosis si se analizan los dientes de erupción tem-
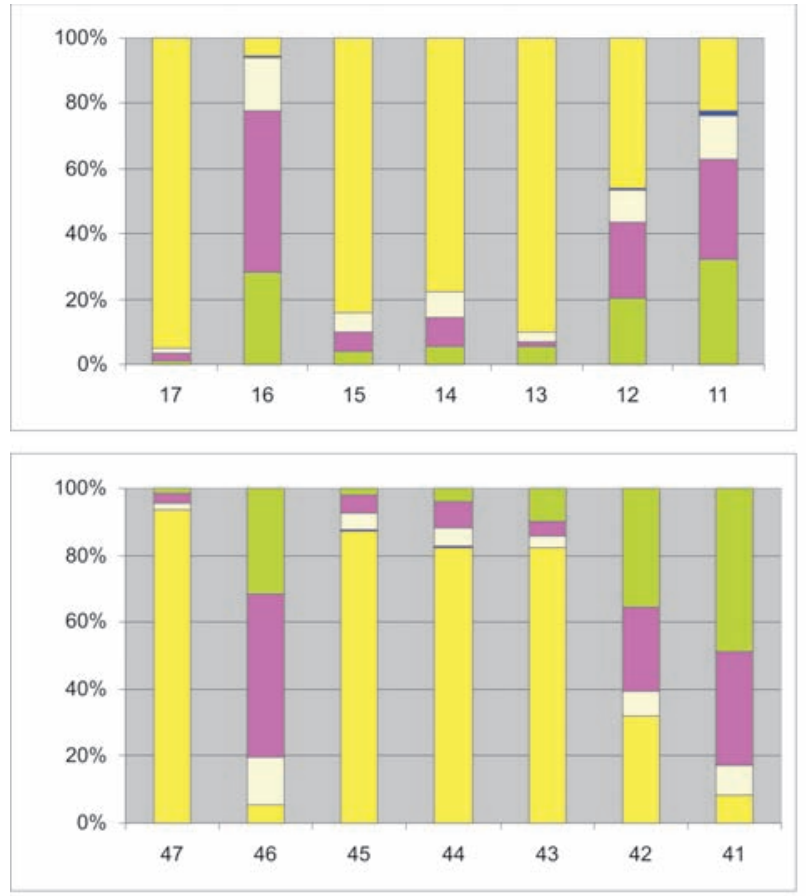

prana (primeros molares e incisivos) o de erupción tardía (caninos y segundos molares). Además, se observó una mayor proporción de dientes excluidos en el examen en los escolares de Oiba si se compara con los de Cepitá. Fueron evidentes mayores niveles de fluorosis en los dientes de erupción temprana en los escolares del municipio de Cepitá comparado con los de Oiba
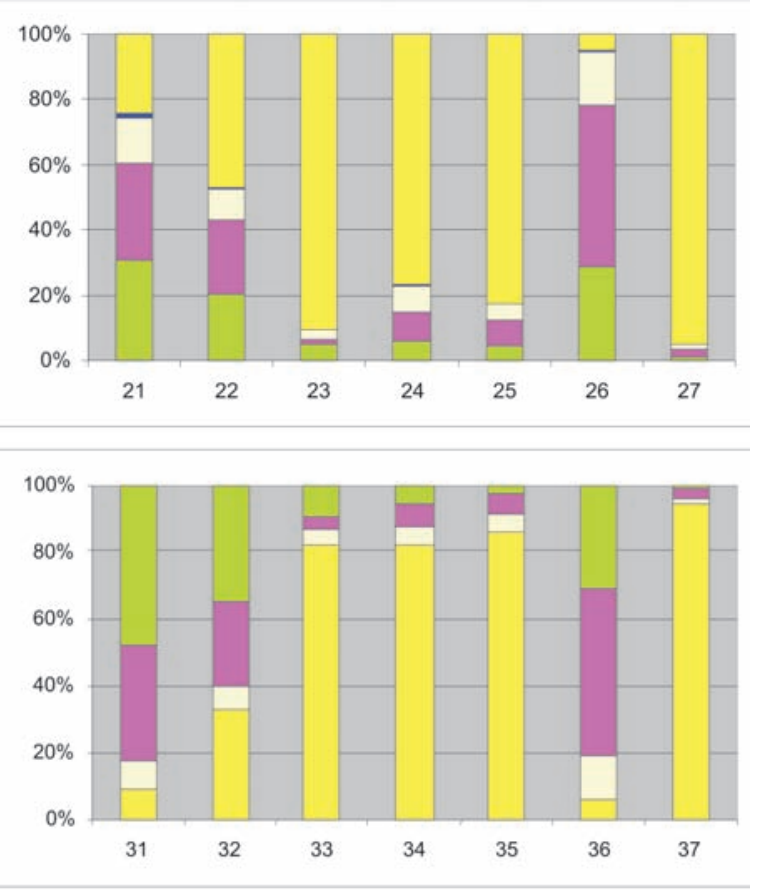

\section{\begin{tabular}{|l|l|l|l|l|}
\hline EXCLUIDO & TFI 5-6 & TFI 3-4 & TFI $1-2$ & TFI 0 \\
\hline
\end{tabular}}

Figura 3. Relación de los niveles de fluorosis en el municipio de Oiba (analizados mediante TFI) según el diente afectado.
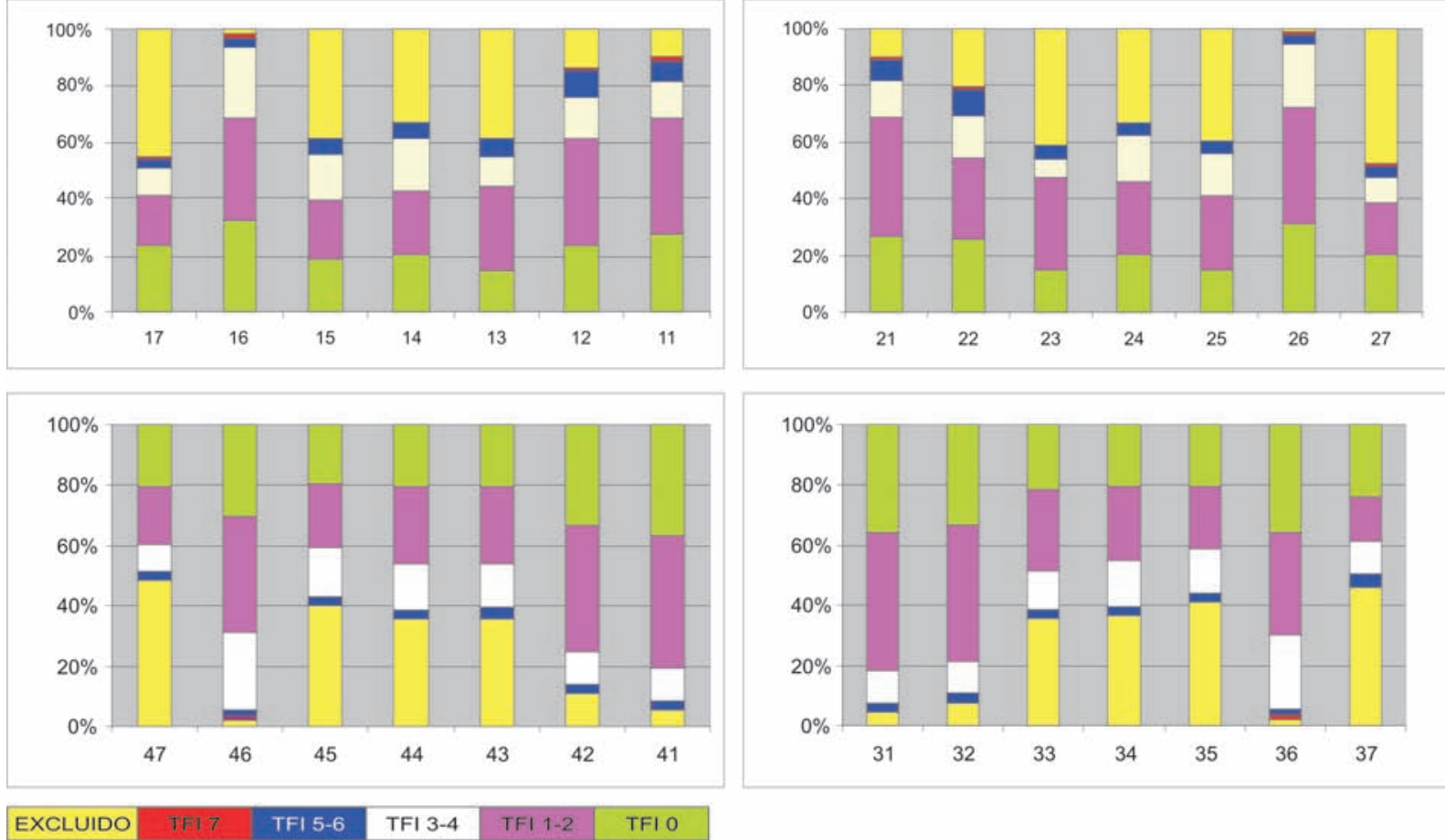

Figura 4. Relación de los niveles de fluorosis en el municipio de Cepitá (analizados mediante TFI) según el diente afectado. 

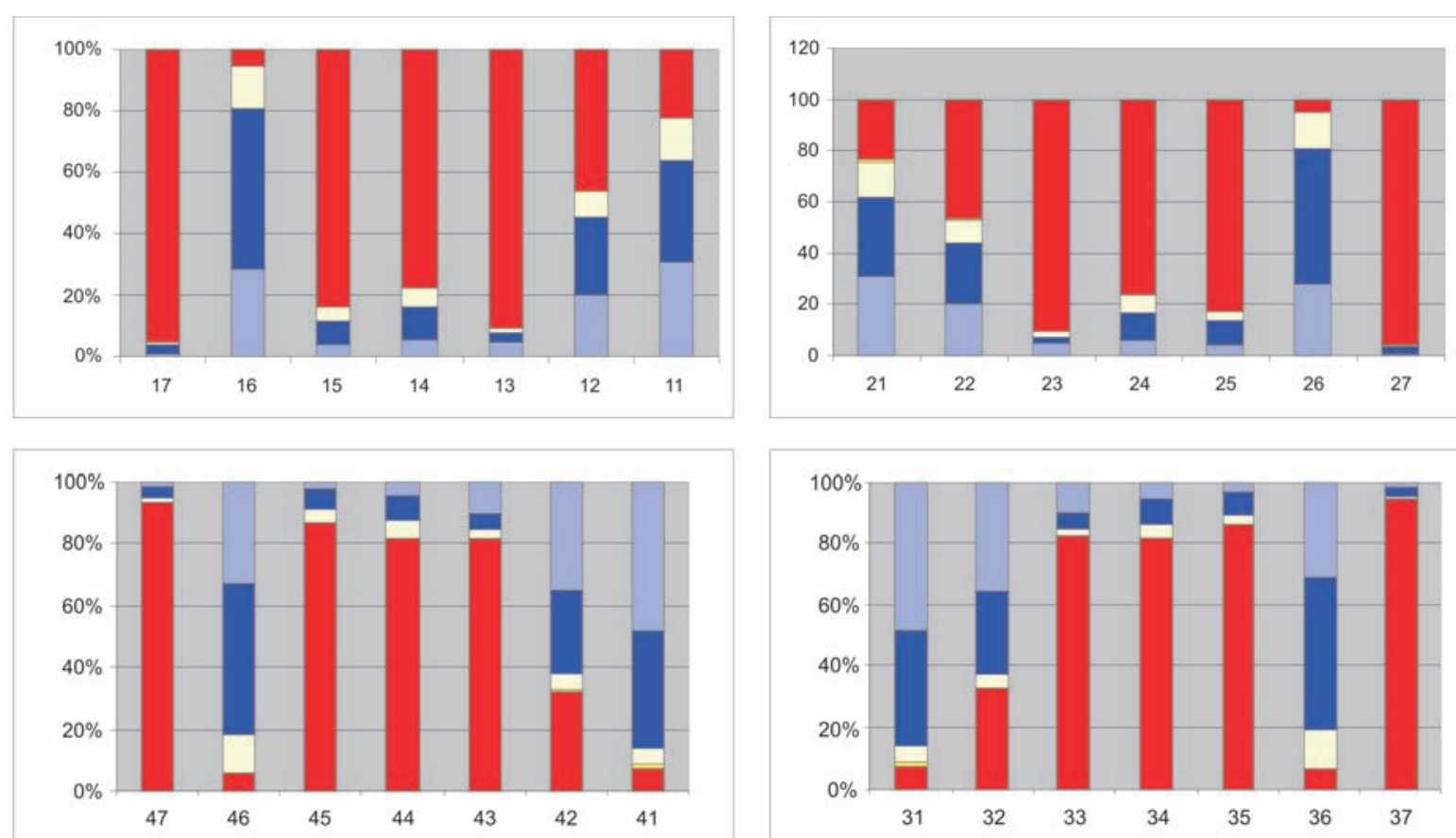

\begin{tabular}{|l|l|l|l|l|}
\hline EXCLUIDO & DEAN 5 & DEAN 3-4 & DEAN 1-2 & DEAN O
\end{tabular}

Figura 5. Relación de los niveles de fluorosis en el municipio de Oiba (analizados mediante el índice de Dean) según el diente afectado.
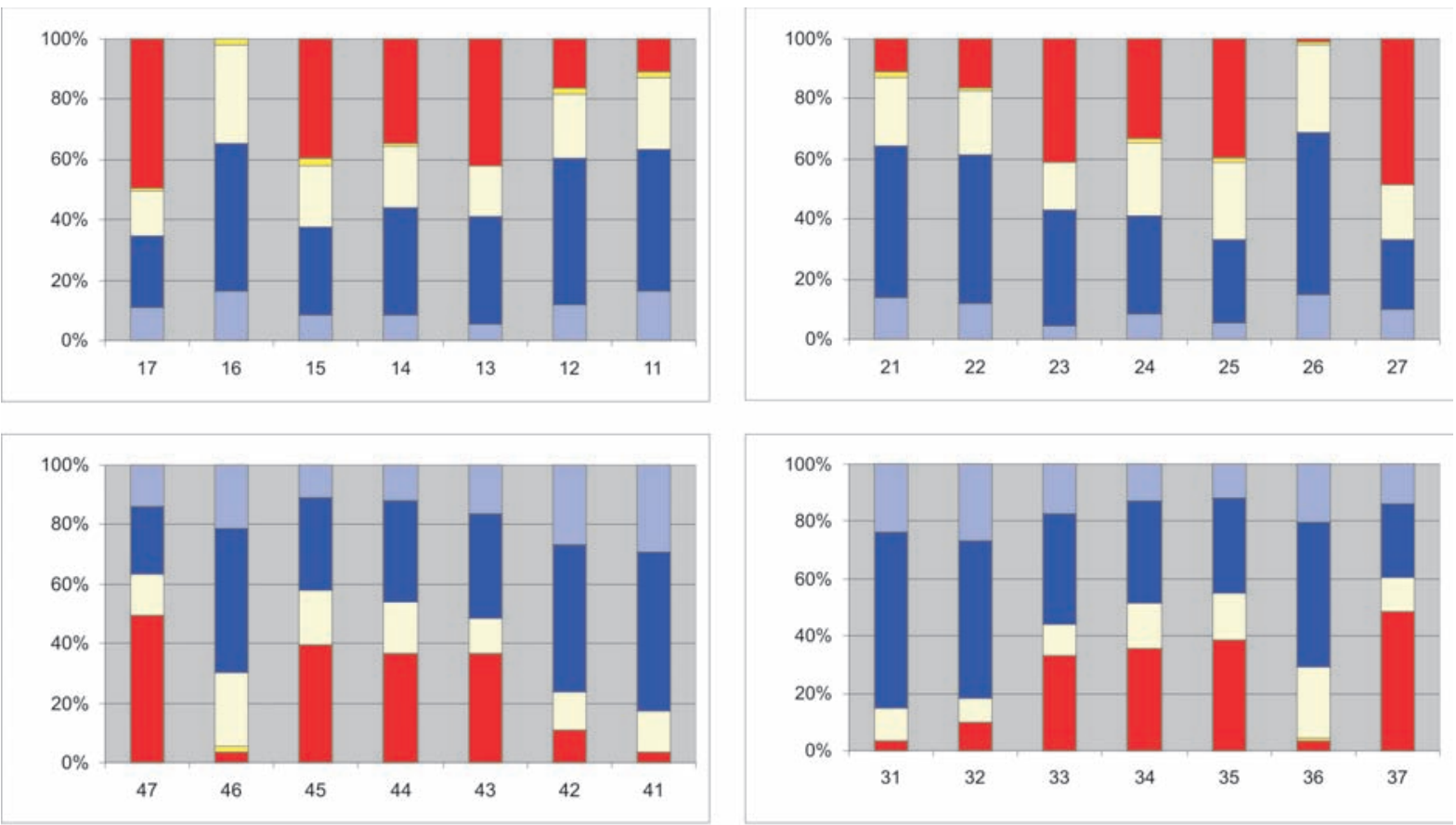

\begin{tabular}{|l|l|l|l|l|} 
EXCLUIDO & DEAN 5 & DEAN 3-4 & DEAN 1-2 & DEAN 0
\end{tabular}

Figura 6. Relación de los niveles de fluorosis en el municipio de Cepitá (analizados mediante el índice de Dean) según el diente afectado. 


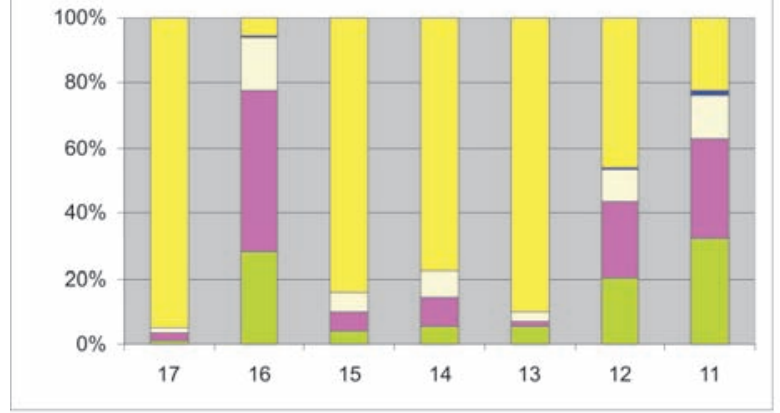

TFI Hemiarcada superior derecha Municipio de Oiba

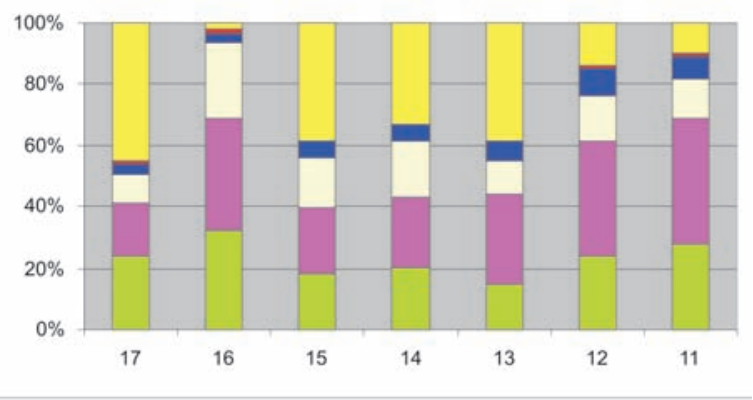

TFI Hemiarcada superior derecha municipio de Cepitá

Figura 7.Comparación de los niveles de TFI en la hemiarcada superior derecha de los municipios de Oiba y Cepitá.

\section{DISCUSIÓN}

El objetivo del presente trabajo fue describir los niveles de fluorosis dental en escolares de los municipios de Oiba y Cepitá, Santander. Se observó una aparente mayor prevalencia y severidad de fluorosis en el municipio de Cepitá. Si se considera lo reportado en el inventario de presencia de flúor en el agua, estudio realizado por el Instituto Nacional de Salud en 1988, en que se registra que en el municipio de Cepitá hay un mayor nivel de este elemento en el agua (0,6 ppm) comparado con la de Oiba $(0,03$ $\mathrm{ppm})$, los resultados parecerían ser consecuentes. ${ }^{22}$

Sin embargo, no se puede desconocer el hecho que las diferencias de edad de los escolares entre los dos municipios podría comprometer la posibilidad de comparar la prevalencia de fluorosis entre estas dos localidades.

Esto obliga a analizar el mejor y peor panorama para Oiba; en el mejor de los casos es probable que el comportamiento de la fluorosis no registre niveles altos de fluorosis; estas especulaciones se fundamentarían en el hecho que las potenciales fuentes de fluoruros no serían diferentes a las registradas por los dientes en erupción temprana por lo cual en los dientes de erupción tardía el comportamiento sería muy similar a los registrados por el otro conjunto de dientes.

En la peor de las suposiciones, cabría esperar que los dientes de erupción tardía registraran mayores niveles y formas más severas de fluorosis; aún en ese caso, se podría pensar que los patrones de fluorosis no alcanzarían a ser similares a los registrados por los escolares del municipio de Cepitá (Figura 7).

Los niveles de fluorosis registrados en los escolares del Municipio de Oiba $(80,7 \%)$ son similares a los registrados en por los escolares del municipio de
Bucaramanga (77,7\%) y Frontino (Antioquia) (67\%) y superior al reportado en el estudio en escolares de Bogotá (48,1\%). ${ }^{3,23,24}$ En el estudio de Frontino se hizo evidente que el problema de fluorosis dental estaba asociado a la llamada multiingesta de flúor que se explica por la sumatoria de las cantidades de flúor ingeridas de los alimentos (preparados con la sal fluorurada) y de la crema dental utilizada para la higiene bucal. ${ }^{3}$ Es probable que para Oiba y las otras ciudades con estas prevalencias la causa sea la misma.

Por su parte los niveles de fluorosis del municipio de Cepitá $(97,8 \%)$ parecen ser similares a los reportados para los municipios de Yondó $(86,7 \%)$ y Sogamoso $(94,6 \%) ;{ }^{3}$ para este último, ${ }^{22}$ el inventario de aguas reporta un nivel de flúor en el agua de $0,06 \mathrm{ppm}$ y no existe reporte para el municipio de Yondó que permita afirmar que la fuente potencial de flúor provenga de las fuentes de agua de consumo, aunque hay reportes informales en los que se hace esta afirmación. ${ }^{25,26} \mathrm{El}$ inventario de agua reporta 0,6 ppm de fluoruros en Cepitá. ${ }^{3}$

Se podria considerar como fortalezas del presente trabajo, la idea de describir el comportamiento de la fluorosis dental en dos municipios en los que se esperaría desigualdades en los niveles de patologías del esmalte asociadas a intoxicación por fluoruros, dada las diferencias potenciales que se podrían suponer en la exposición a los fluoruros, como un mecanismo de monitoreo del comportamiento de la fluorosis y una estrategia para hacer vigilancia epidemiológica; más aún, si no se evidencian en la literatura revisada otras fuentes que permitan comparar en Colombia, el comportamiento actual de la fluorosis con información pasada (años 60 ó 70).

En términos prácticos, el presente trabajo ilustra sobre la importancia de evaluar esta condición en 
escolares en edades en las que la exclusión dentaria por ausencia o procesos de erupción permita cuestionar los resultados; fundamentados en la información recolectada, se propone analizar el comportamiento de la fluorosis en escolares entre 12 y 17 años pues de esta manera se reduce la cantidad de dientes que podrían ser excluidos.

Para terminar es importante resaltar el potencial impacto que puede tener la fluorosis sobre aspectos relacionados con la autoimagen que los escolares perciben, pues aunque algunos autores reportan que quizás éste no sea el aspecto más relevante, ${ }^{27}$ no se puede desconocer que para los adolescentes este es un aspecto muy importante y que determina las acciones que la profesión odontológica debe asumir en miras de resolver esta situación; ya que la presencia y severidad de la fluorosis parece estar determinado el nivel de insatisfacción de los escolares frente a la apariencia de sus dientes, evidenciándose en el hecho que cerca del 68\% de los alumnos de Cepitá (municipio con mayor nivel de fluorosis) manifestaron esta insatisfacción comparado con el $6,8 \%$ de los estudiados de Oiba.

Se concluye que la prevalencia observada en el municipio de Cepitá $(97,8 \%)$ y en Oiba del $(80,7 \%)$, los resultados no podrían compararse ya que las diferencias de edad entre las poblaciones y en la cronología de la erupción, podrían explicar las diferencias observadas.

\section{BIBLIOGRAFÍA}

1. Azpeitia-Valadez ML, Rodríguez-Frausto M, Sánchez-Hernández MA. Prevalencia de fluorosis dental en escolares de 6 a 15 años de edad. Rev Med Inst Mex Seguro Soc 2008; 46: $67-72$.

2. Cury JA, Machado Tabchoury CP. Determination of appropriate exposure to fluoride in non-EME countries in the future. J Appl Oral Sci 2003; 11: 83 - 95.

3. Ramírez BS, Franco AM, Sierra JL, López RV, Alzate T, Sarrázola AM. Fluorosis dental en escolares y exploración de factores de riesgo. Municipio de Frontino, 2003. Revista Facultad de Odontología Universidad de Antioquia 2006; 17: $26-33$.

4. Soto-Rojas AE, Ureña-Cirett JL, Martínez-Mier EA. A review of the prevalence of dental fluorosis in Mexico. Rev Panam Salud Pública. 2004; 15: 9 - 18.

5. Martínez Herrera E, Agudelo Suárez AA, Viña Sarmiento Y, Duque Gómez LF, Tobón Cifuentes MM. Alcances y limitaciones del flúor dentro de la política pública en salud bucal en Colombia. Rev Gerenc Polit Salud 2007; 6: $34-$ 44.

6. Sosa Rosales MC, García Melian M, Gómez A, González I, Mojáiber de la Peña A. Sistema de vigilancia para el Programa de Fluoruración de la Sal de consumo Humano en Cuba. Rev Cubana Salud Pública [en línea].2004 Dic [Fecha de acceso: 12 de febrero de 2008]; 30: URL disponible en: http:// scielo.sld.cu/scielo.php?script $=$ sci arttext\&pid $=$ S0864$34662004000400011 \& \operatorname{lng}=\mathrm{es} \& \mathrm{nrm}^{-}=$iso
7. Fejerskov O, Manji F, Baelum V, Moller IJ. Dental Fluorosis. A Handbook for Health Worker. Copenhagen: Munksgaard; 1988.

8. República de Colombia, Ministerio de Salud, Centro Nacional de Consultoría (CNC). Estudio Nacional de Salud Bucal. En: Ministerio de Salud - República de Colombia, Centro Nacional de Consultoría - CNC: III Estudio Nacional de Salud Bucal - ENSAB III; Tomo VII. Bogotá: Lito Servicios ALER; 1999.

9. Tovar S. Dentro de los compromisos de la odontología en la salud pública: vigilancia del uso de los fluoruros. Revista Federación Odontológica Colombiana 2001; 201: 33 - 47.

10. República de Colombia. Ministerio de Protección Social. Resolución 3577, Septiembre 2006.

11. República de Colombia Ministerio de Protección Social. Decreto 3039 de Agosto de 2007.

12. República de Colombia. Ministerio de Protección Social. Resolución 0425 de 2008.

13. Moncada OA, Jiménez G. La sal alimento enriquecido para la prevención de la salud en Colombia. Revista Federación Odontológica Colombiana 1997; 192: 34 - 38.

14. Fenstein AR. Clinical biostatistics. XLII. The architecture of cross-sectional research (Part I). Clinic Pharmacol Ther 1978; 23: 81 - 96.

15. Fenstein AR. Clinical biostatistics. XLIII. The architecture of cross-sectional research (conclusion). Clinic Pharmacol Ther 1978; 23: 481 - 493.

16. Microsoft Excel 5.0. NY. Microsoft Corporation, 1997.

17. CDC-OMS. Epi-info 6, Versión 6.04. Atlanta, (Nov 1996).

18. STATA Corp. Stata Statistical Software. Release 8.0. College station. TX: Stata corporation 2003.

19. Pagano M, Gauvreau K. Principles of Biostatistics. Belmont: Duxbury Press. (1995) p. 35 - 44, 257 - 263.

20. Norman GR, Streiner DL. Bioestadística. Madrid:Mosby/ Doyma Libros, 1996. p. 129-142.

21. República de Colombia. Ministerio de Salud. Resolución No. 008430 (octubre 4 de 1993), Bogotá.

22. Moncada OA, Jiménez G. Inventario del contenido natural de flúor en las aguas para consumo público. Revista Federación Odontológica Colombiana 1995; 51: 17 - 26.

23. Martignon S, Granados OL. Prelavencia de fluorosis dental y análisis de asociación a factores de riesgo en escolares de Santafé de Bogotá. Universidad El Bosque. División de Posgrados. Santafé de Bogotá; 1999.

24. Concha S, Celedón Y, Vera W, Poveda E, Muñoz C, Vergel T, Luna D, Rodríguez L, Arteaga C, Camargo DM. Prevalencia de fluorosis dental en escolares de 6 a 15 años de edad de la zona urbana de Bucaramanga. Ustasalud Odontología 2003; 2: $73-82$.

25. Distritos Mineros - Línea de Base de Ordenamiento Territorial. URL disponible en: http://distritosmineros.gov. co/apps/ 't/files/puertonare/perfilsubregionalmagdalenamedio.doc

26. Televisión Regional. Programa: Hablemos de Salud. Referencia: Fluorosis Yondó Emisión Septiembre 17 de 2005. URL disponible en: http://www.dssa.gov.co/htm/ prog2005,htm

27. Maura E, McPherson L, Simmons DR, Gilmour H, Stephen K. An assessment of teenagers' perceptions of dental fluorosis using digital simulation and web-based testing. Community Dent Oral Epidemiol 2005; 33: 298 - 306. 